TRANSACTIONS OF THE

AMERICAN MATHEMATICAL SOCIETY

Volume 352, Number 6, Pages $2913-2936$

S 0002-9947(00)02409-0

Article electronically published on March 2, 2000

\title{
INFINITE CONVOLUTION PRODUCTS AND REFINABLE DISTRIBUTIONS ON LIE GROUPS
}

\author{
WAYNE LAWTON
}

\begin{abstract}
Sufficient conditions for the convergence in distribution of an infinite convolution product $\mu_{1} * \mu_{2} * \ldots$ of measures on a connected Lie group $\mathcal{G}$ with respect to left invariant Haar measure are derived. These conditions are used to construct distributions $\phi$ that satisfy $T \phi=\phi$ where $T$ is a refinement operator constructed from a measure $\mu$ and a dilation automorphism $A$. The existence of $A$ implies $\mathcal{G}$ is nilpotent and simply connected and the exponential map is an analytic homeomorphism. Furthermore, there exists a unique minimal compact subset $\mathcal{K} \subset \mathcal{G}$ such that for any open set $\mathcal{U}$ containing $\mathcal{K}$, and for any distribution $f$ on $\mathcal{G}$ with compact support, there exists an integer $n(\mathcal{U}, f)$ such that $n \geq n(\mathcal{U}, f)$ implies $\operatorname{supp}\left(T^{n} f\right) \subset \mathcal{U}$. If $\mu$ is supported on an $A$-invariant uniform subgroup $\Gamma$, then $T$ is related, by an intertwining operator, to a transition operator $W$ on $\mathbb{C}(\Gamma)$. Necessary and sufficient conditions for $T^{n} f$ to converge to $\phi \in L^{2}$, and for the $\Gamma$-translates of $\phi$ to be orthogonal or to form a Riesz basis, are characterized in terms of the spectrum of the restriction of $W$ to functions supported on $\Omega:=\mathcal{K} \mathcal{K}^{-1} \cap \Gamma$.
\end{abstract}

\section{Introduction and Statement of Results}

This paper extends concepts, associated with the theory of refinable functions and wavelets on $\mathbb{R}^{d}$, to a connected Lie group $\mathcal{G}$.

In $\$ 2$ we consider a sequence of measures $\mu_{i}$, whose integrals equal 1 , whose total variations $\left|\mu_{i}\right|$ are uniformly bounded, and whose $\operatorname{supports} \operatorname{supp}\left(\mu_{i}\right)$ converge exponentially fast to the identity $1 \in \mathcal{G}$. Define convolution products

$$
\Phi_{n}:=\mu_{1} * \mu_{2} * \mu_{3} * \ldots * \mu_{n}
$$

with respect to left-invariant Haar measure $d g$ on $\mathcal{G}$. The major result in this paper is Theorem 2.1 which shows that there exists a compactly supported distribution $\phi$ on $\mathcal{G}$ such that

$$
\lim _{n \rightarrow \infty} \Phi_{n}=\phi .
$$

For an abelian group $\mathcal{G}$ this result is standard ([11], p. 176), [12], and is obtained by applying the Paley-Wiener-Schwartz theorem [50] to the infinite product formula

Received by the editors March 10, 1997 and, in revised form, April 9, 1998.

1991 Mathematics Subject Classification. Primary 41A15, 41A58, 42C05, 42C15, 43A05, $43 \mathrm{~A} 15$.

Key words and phrases. Lie group, distribution, enveloping algebra, dilation, refinement operator, cascade sequence, transition operator, condition E, Riesz basis.

Research supported in part by the NUS Wavelets Program funded by the National Science and Technology Board and the Ministry of Education, Republic of Singapore. 
for the Fourier transform

$$
\widehat{\phi}(y)=\lim _{n \rightarrow \infty} \prod_{i=1}^{n} \widehat{\mu}_{i}(y) .
$$

For non-abelian groups the proof proceeds from a series of lemmas that describe the effect of right convolution by $\mu_{i}$ on the right-invariant derivatives of a function. The remaining sections derive properties of $\phi$ under more restricted assumptions on the sequence $\mu_{i}$.

In $\$ 3$ we consider an automorphism $A$ of $\mathcal{G}$ whose derivative at 1 has all eigenvalues with modulus $>1$. Denote the Lie algebra of $\mathcal{G}$, identified with the tangent space to $\mathcal{G}$ at 1 , by $\mathcal{L}$. The automorphism $A$ is called a dilation and its derivative at 1 (also denoted by $A$ ) is an automorphism of $\mathcal{L}$. The existence of a dilation imposes conditions on $\mathcal{G}$. Lemma 3.1 shows $\mathcal{G}$ is a nilpotent group. This follows directly from a theorem of Jacobson [27. We provide a proof that illustrates the relationship between the descending central series for $\mathcal{L}$ and the invariant subspaces of $A$. Lemma 3.2 shows $\mathcal{G}$ is simply connected. Therefore, a result of Malcev implies the exponential map is an analytic homeomorphism of $\mathcal{L}$ onto $\mathcal{G}$. Theorem 3.1 shows $A^{-1}$ is a contraction with respect to a suitably chosen left-invariant metric on $\mathcal{G}$.

In $₫ 4$ we consider general refinement operators. Let $A$ be a dilation and denote the determinant of $A$ by $|A|$. The dilation operator $D$ is defined on distributions by

$$
\langle D f, h\rangle:=\left\langle f, h\left(A^{-1} \cdot\right)\right\rangle,
$$

where $f$ is a distribution, $h$ is a test function, and $\langle f, h\rangle$ denotes the value of the distribution $f$ at $h$. Therefore, if $f$ is a function,

$$
(D f)(x)=|A| f(A x) .
$$

A refinement mask is a measure $\mu$ whose integral equals 1 , whose total variation $|\mu|$ is finite, and whose support $\operatorname{supp}(\mu)$ is compact. The $(A, \mu)$-refinement operator $T$ is defined on distributions by

$$
T f:=D(\mu * f) \text {. }
$$

A distribution $\phi$ is $(A, \mu)$-refinable if $\phi$ is nonzero, $\operatorname{supp}(\phi)$ is compact, and $\phi$ satisfies the refinement equation $T \phi=\phi$. Since left invariant Haar measure satisfies $d(A g)=|A| d g$, the sequence $\mu_{i}=D^{i} \mu$ is a sequence of refinement masks that satisfies the hypothesis of Theorem 2.1 Clearly the cascade sequence $T^{n} f$ satisfies

$$
T^{n} f=\Phi_{n} * D^{n} f
$$

where $\Phi_{n}$ is defined by (1.1). Theorem 4.1 shows for any compactly supported distribution $f$ whose integral is nonzero, the cascade sequence $T^{n} f$ converges in distribution to a refinable distribution $\phi$ that is unique up to a scalar multiple. Theorem 4.2 shows there exists a unique minimal compact subset $\mathcal{K} \subset \mathcal{G}$ such that for any open set $\mathcal{U}$ containing $\mathcal{K}$, and for any distribution $f$ on $\mathcal{G}$ having compact support, there exists an integer $n(\mathcal{U}, f)$ such that $n \geq n(\mathcal{U}, f)$ implies $\operatorname{supp}\left(T^{n} f\right) \subset \mathcal{U}$.

In $\$ 5$ we consider discrete refinement operators defined as $(A, \mu)$-refinement operators whose refinement mask $\mu$ is supported on an $A$-invariant uniform subgroup $\Gamma \subset \mathcal{G}$ ( $\Gamma$ is discrete and the quotient space, consisting of right cosets $\Gamma \backslash \mathcal{G}$, is a compact manifold). Therefore the refinement mask $\mu$ can be expressed in the form $\mu_{c}:=\sum_{\gamma \in \Gamma} c(\gamma) \delta_{\gamma}$, where $\delta_{\gamma}$ denotes the point measure at $\gamma$, and where $c$ is a 
finitely supported complex-valued function $c$ on $\Gamma$ that satisfies $\sum_{\gamma \in \Gamma} c(\gamma)=1$. We call the function $c$ a refinement mask and we let $T_{c}$ denote the $\left(A, \mu_{c}\right)$-refinement operator (or $(A, c)$-refinement operator). Let $\Gamma_{o} \subset \Gamma$ contain 1 and form a complete set of representatives for the right cosets $A \Gamma \backslash \Gamma$. Given a refinement mask $c$, for $r \in \Gamma$ define

$$
c_{r}:=\sum_{\gamma \in \Gamma} c((A \gamma) r)
$$

Clearly $c_{r}$ depends only on the $\operatorname{coset}(A \Gamma) r$. A refinement mask $c$ is fundamental if

$$
c_{r}=|A|^{-1}, \quad r \in \Gamma .
$$

Let $\chi_{\Gamma_{o}}$ denote the characteristic function of $\Gamma_{o}$ and define $d:=|A|^{-1} \chi_{\Gamma_{o}}$. Lemma 5.1 shows that $\operatorname{card}\left(\Gamma_{o}\right)=|A|$, hence $d$ is a refinement mask. Lemma 5.2 constructs a tile $\mathcal{T} \subset \mathcal{G}$ whose characteristic function is $\left(A, \mu_{d}\right)$-refinable. For $g \in \mathcal{G}$, we define the left-translation operator on distributions by $\left\langle L_{g} f, h\right\rangle:=\langle f, h(g \cdot)\rangle$, and observe that if $f$ is a function, then $\left(L_{g} f\right)(x)=f\left(g^{-1} x\right)$. We call a distribution $f$ a Schoenberg distribution if $\sum_{\gamma \in \Gamma} L_{\gamma} f=1$. Schoenberg gave a characterization of these and related distributions on $\mathbb{R}$ [4], while Strang and Fix later discussed their approximation properties in the context of finite element methods [51]. The characteristic function of any fundamental domain for $\Gamma \backslash \mathcal{G}$ is a Schoenberg distribution. Theorem 5.1 shows $T_{c} f$ is a Schoenberg distribution whenever $c$ is a fundamental refinement mask and $f$ is a Schoenberg distribution. Corollary 5.1 shows that if $c$ is fundamental, then any $\left(A, \mu_{c}\right)$-refinable distribution is proportional to a Schoenberg distribution. Lemarie used variational methods to construct multiresolution analyses on stratified Lie groups [38. The associated refinable functions are infinitely supported regular splines that interpolate $\delta_{1} \in \mathbb{C}(\Gamma)$ and minimize a functional constructed from a hypo-elliptic sub-Laplacian operator. In contrast, the refinable distributions $\phi$ constructed in this paper have compact support and, in general, may not be regular.

In $\$ 6$ we discuss the relationship between conditions for the cascade sequence to converge strongly to $\phi \in L^{2}(\mathcal{G})$ and properties of a transition operator constructed from $A$ and $c$. Let $A$ be a dilation of $\mathcal{G}$, let $\Gamma \subset \mathcal{G}$ be an $A$-invariant uniform subgroup, and let $c$ be a refinement mask supported on $\Gamma$. Define the $(A, c)$-transition operator $W: \mathbb{C}(\Gamma) \rightarrow \mathbb{C}(\Gamma)$, by

$$
(W b)(\gamma):=\sum_{\alpha \in \Gamma} K(\gamma, \alpha) b(\alpha)
$$

where

$$
K(\gamma, \alpha):=\sum_{\beta \in \Gamma}|A| c\left((A \gamma) \beta \alpha^{-1}\right) \overline{c(\beta)} .
$$

The formal adjoint of $W$ is the subdivision operator $S$, thus

$$
(S b)(\gamma)=\sum_{\alpha \in \Gamma} \overline{K(\alpha, \gamma)} b(\alpha)
$$

For $\mathcal{G}$ abelian and $A=2 I$, the subdivision operator was studied in [4], [15], [29] in the context of curve and surface approximation, while the transition operator $W$ was studied in [6], [7], [10], [32], [33], [34], [36], [37], [57] in the context of wavelets. This paper extends related concepts to the context of nonabelian groups. 
Theorem 6.1 shows that a mask $c$ is fundamental if and only if the constant function $1 \in \mathbb{C}(\Gamma)$ is an eigenvector of $S$ having eigenvalue 1 . For any distribution $f$ and test function $h$, define $\langle\tilde{f}, h\rangle:=\langle f, \Delta \tilde{h}\rangle$ where $\tilde{h}(g):=\overline{h\left(g^{-1}\right)}$ and $\Delta$ is the modular function on $\mathcal{G}$. Therefore for a function $f, \tilde{f}(g)=\overline{f\left(g^{-1}\right)}$. Define the operator $[\cdot, \cdot]: L^{2}(\mathcal{G}) \times L^{2}(\mathcal{G}) \rightarrow \mathbb{C}(\Gamma)$ by $[f, h](\gamma):=(f * \tilde{h})(\gamma)$. Since $f * \tilde{h} \in L^{2}(\mathcal{G})$, its restriction to $\Gamma$ is well defined. Theorem 6.2 shows that

$$
[T f, T h]=W([f, h]) .
$$

Let $\mathcal{K} \subset \mathcal{G}$ be the compact subset in Theorem 4.2 and define the finite subset $\Omega \subset \Gamma$ by

$$
\Omega:=\mathcal{K K}^{-1} \cap \Gamma .
$$

Theorem 6.3 shows for every finitely supported $b \in \mathbb{C}(\Gamma)$, there exists an integer $n(b)$ such that $n \geq n(b)$ implies $\operatorname{supp}(W b) \subset \Omega$. Furthermore, $\Omega$ is the minimal set with this property. Define the restricted transition operator $W_{r}$ to be the restriction of $W$ to functions supported on $\Omega$. If $\mathcal{G}$ is abelian, the eigenvalues of $W_{r}$ describe many important properties of the refinement operator $T$ and the corresponding refinable distribution $\phi$, including convergence of the cascade algorithm, regularity of $\phi$, and whether the $\Gamma$-translates of $\phi$ form an orthonormal or a Riesz basis, (see [5], [6], [7], [7], [16], [23], [32], 33], [34, 36], 37], 48]). For the case $\mathcal{G}=R$, and $A=2$, it was shown in [33] and [34] that if $W_{r}$ has eigenvector $\delta_{0}$ with eigenvalue 1 , then $\phi \in L^{2}(\mathbb{R})$. Furthermore, it was shown that the integer translates of $\phi$ are orthogonal if and only if 1 is a simple eigenvalue of $W_{r}$. These results were extended to general abelian groups and general dilations in [35], [36] and [37, to vector-valued refinable functions in 48. We summarize below our extensions of these results to general Lie groups. An operator satisfies condition $E$ if 1 is a simple eigenvalue and all other eigenvalues have modulus $<1$. An operator satisfies restricted condition $\mathrm{E}$ if all eigenvalues have modulus $\leq 1$ and all eigenvalues having modulus 1 are nondegenerate. Theorem 6.4 shows that if $W_{r}$ satisfies extended condition E, then for any $f \in L^{2}(\mathcal{G})$ the cascade sequence $T^{n} f$ converges weakly in $L^{2}(\mathcal{G})$. This result extends Proposition 3.1 in 37 . Theorem 6.5 shows that the cascade sequence $T^{n} f$ converges strongly in $L^{2}(\mathcal{G})$ for every Schoenberg function $f \in L^{2}(\mathcal{G})$ if and only if $W_{r}$ satisfies condition $\mathrm{E}$ and $c$ is fundamental. This result extends Theorem 2.2 in 37. Theorem 6.6 shows that if a function $\phi \in L^{2}(\mathcal{G})$ is refinable, and if $[\phi, \phi] \in \mathbb{C}(\Omega)$ has a convolution inverse in $\ell^{1}(\Gamma)$, then $W_{r}$ satisfies condition $\mathrm{E}$. If $\mathcal{G}$ is abelian, Wiener's lemma implies this hypothesis is equivalent to stability of $\phi$. Therefore Theorem 6.6 can be interpreted as an extension of Theorem [2.1] in [36].

This paper is limited to developing a foundation for a theory of compactly supported orthonormal and biorthogonal wavelets on Lie groups. It does not address the problem of constructing smooth refinable distributions, nor does it address the problem of constructing wavelet bases. However, we note that Bagget, Carey, Moran, and Ohring showed [1], using properties of von Neumann algebras [19], [9], that the existence of stable refinable functions in $L^{2}(\mathcal{G})$ implies the existence of orthonormal wavelet bases for $L^{2}(\mathcal{G})$.

\section{Infinite Convolution Products}

2.1. Preliminaries. Let $\mathbb{N}, \mathbb{Z}, \mathbb{R}, \mathbb{C}$ denote the nonnegative integers, the discrete group of integers, and the locally compact fields of real and complex numbers, respectively. Let $\mathcal{G}$ denote a connected Lie group with identity 1 , dimension $d \geq 1$, 
and left-invariant Haar measure $d g$. The modular function $\Delta: \mathcal{G} \rightarrow R$ defined by $d\left(g x^{-1}\right):=\Delta(x) d g$ is an analytic homomorphism of $\mathcal{G}$ into the group of positive real numbers that satisfies $\int f(g x) d g=\Delta(x) \int f(g) d g$ and $d g^{-1}=\Delta(g) d g$. Define topological vector spaces of complex-valued functions on $\Gamma$ and $\mathcal{G}$ and distributions on $\mathcal{G}$ as follows:

(a) Banach spaces $\ell^{p}(\Gamma)$ and $L^{p}(\mathcal{G}), 1 \leq p \leq \infty$, and their duals $\ell^{q}(\Gamma)$ and $L^{q}(\mathcal{G})$, for $p<\infty$, where $\frac{1}{p}+\frac{1}{q}=1$.

(b) Banach space $\mathbb{C}^{N}(\mathcal{G})$ of complex-valued $C^{N}$-regular functions, with topology of uniform convergence of derivatives up to order $N$ over compact subsets, and the dual space $\mathcal{M}(\mathcal{G})$ of $\mathbb{C}^{0}(\mathcal{G})$, consisting of compactly supported complex-valued Borel measures $\mu$ whose total variation $|\mu|<\infty$. Let $\mathbb{C}_{c}^{N}(\mathcal{G})$ denote the subspace of $\mathbb{C}^{N}(\mathcal{G})$ consisting of functions having compact support.

(c) Banach subspace $\mathbb{C}^{N}(\mathcal{K}) \subset \mathbb{C}_{c}^{N}(\mathcal{G})$ consisting of functions supported on a compact subset $\mathcal{K} \subset \mathcal{G}$.

(d) Fréchet space $\mathcal{E}(\mathcal{G})$ consisting of infinitely differentiable complex-valued functions on $\mathcal{G}$, with topology of uniform convergence of derivatives of finite order over compact subsets, and its dual space $\mathcal{E}^{\prime}(\mathcal{G})$ of compactly supported distributions.

(e) Test space $\mathcal{D}(\mathcal{G})$ consisting of compactly supported $f \in \mathcal{E}(\mathcal{G})$ with the inductive limit topology, and its dual space $\mathcal{D}^{\prime}(\mathcal{G})$ consisting of distributions.

The inductive limit topology is defined in ([41, p. 69) and in (44, p. 37). Distribution spaces are also discussed in [20], [52], [55] and [58. For any vector $v$ in one of these spaces and vector $w$ in its dual space, let $\langle w, v\rangle$ denote the value of the linear functional $w$ at $v$. Define convolution of functions by

$$
f * h(y):=\int f(x) h\left(x^{-1} y\right) d x
$$

and extend to distributions. Then $\operatorname{supp}(f * h)=\{x y \mid x \in \operatorname{supp}(f), y \in \operatorname{supp}(h)\}$. Let $\mathcal{L}$ denote the Lie algebra of $\mathcal{G}$ identified with the tangent space at 1 . Define the exponential map exp : $\mathcal{L} \rightarrow \mathcal{G}$ so $t \rightarrow \exp (t X)$ is the unique analytic homomorphism from $\mathbb{R}$ into $\mathcal{G}$ whose derivative at $t=0$ equals $X$. Then exp is an analytic homeomorphism of some open neighborhood $U$ of $0 \in \mathcal{L}$ onto some open neighborhood $V$ of $1 \in \mathcal{G}$. Let $\log : V \rightarrow U$ denote the inverse function of $\left.\exp \right|_{U}$.

For $g \in \mathcal{G}$ the automorphism $\operatorname{Ad}(g): \mathcal{L} \rightarrow \mathcal{L}$ is defined as the derivative of the inner automorphism $x \rightarrow g x g^{-1}$ on $\mathcal{G}$ at $x=1$. Thus $\exp (A d(g) X)=g \exp (X) g^{-1}$ and $|A d(g)|=\Delta(g)$.

Let $\mathcal{U}$ denote the right enveloping algebra, consisting of right-invariant analytic differential operators on $\mathcal{G}$, with the Lie product

$$
\left[U_{1}, U_{2}\right]:=U_{1} U_{2}-U_{2} U_{1} \text {. }
$$

For $X \in \mathcal{L}$ define the right-invariant differential operator

$$
(D(X) f)(g):=\lim _{t \rightarrow 0} \frac{f(\exp (t X) g)-f(g)}{t},
$$

Then $[D(X), D(Y)]=-D([X, Y])$, see $\left([54]\right.$, p. 12). Fix a basis $\left\{X_{1}, \ldots, X_{d}\right\}$, define $D_{i}:=D\left(X_{i}\right)$, and for any multi-integer $\mathbf{n}=\left(n_{1}, \ldots, n_{d}\right) \in \mathbb{N}^{d}$ define the monomial $D^{\mathbf{n}}:=D_{1}^{n_{1}} \ldots D_{d}^{n_{d}}$ (where $D_{i}^{0}:=I$ ). The Poincaré-Birkhoff-Witt theorem, [8], [25], [26], [28], [54], [56], implies that the monomials form a basis for $\mathcal{U}$. 
Define $|\mathbf{n}|:=\sum_{k} n_{k}$ and for $p \in N$ define

$$
\mathcal{U}^{p}:=\left\{\sum_{|\mathbf{n}| \leq p} a(\mathbf{n}) D^{\mathbf{n}}: a: \mathbb{N}^{d} \rightarrow \mathbb{C}\right\} .
$$

Then $\mathcal{U}^{p}$ is independent of the choice of basis and consists of all analytic differential operators of degree $\leq p$.

For $N \in \mathbb{N}$, define the norm $\|\cdot\|_{N}$ on $\mathbb{C}_{c}^{N}(\mathcal{G})$ by

$$
\|h\|_{N}:=\max _{g \in \mathcal{G}}\left[\sum_{|\mathbf{n}| \leq N}\left|\left(D^{\mathbf{n}} h\right)(g)\right|^{2}\right]^{\frac{1}{2}} .
$$

Clearly, this norm defines the topology on $\mathbb{C}^{N}(\mathcal{K})$ for any compact subset $\mathcal{K}$ of $\mathcal{G}$.

2.2. Derivations. Let $\mathcal{M}$ be a $C^{1}$ manifold having dimension $d$ and let $0<\alpha<1$. A sequence $\mathcal{M}_{n} \subset \mathcal{M}$ converges exponentially fast at rate $\alpha$ to $u \in \mathcal{M}$ if there exists an open subset $U \subset \mathcal{M}$ containing $u$, a $C^{1}$ homeomorphism $F$ from $U$ onto an open subset $V \subset \mathbb{R}^{d}$ such that $F(u)=0$, a vector norm $\|\cdot\|$ on $\mathbb{R}^{d}$, and a constant $C>0$, such that for all $v_{n} \in \mathcal{M}_{n}$

$$
\left\|F\left(v_{n}\right)\right\| \leq C \alpha^{n} .
$$

This definition is independent of the homeomorphism and the vector norm. Let $\rho: \mathcal{G} \times \mathcal{G} \rightarrow \mathbb{R}$ be any left-invariant metric, [2], 31]. For $G \subset \mathcal{G}$ define

$$
\rho(G):=\sup \{\rho(g, 1): g \in G\} .
$$

Then the triangle inequality for norms implies

$$
\rho(\operatorname{supp}(f * h)) \leq \rho(\operatorname{supp}(f))+\rho(\operatorname{supp}(h)) .
$$

An inner product $(\cdot, \cdot)$ on $\mathcal{L}$ induces a left-invariant Riemannian structure on the tangent bundle $T(\mathcal{G})$ and therefore defines a left-invariant geodesic metric $\rho$. A sequence $G_{i} \subset \mathcal{G}$ is uniformly bounded if there exists a compact $G \subset \mathcal{G}$ such that $G_{i} \subset G$. This property is equivalent to boundedness of the sequence $\rho\left(G_{i}\right)$ for some (any) left-invariant geodesic metric $\rho$.

Lemma 2.1. Let $\mu_{i} \in \mathcal{M}(\mathcal{G})$ such that supp $\left(\mu_{i}\right)$ converges exponentially fast to 1 . Define $\Phi_{n}:=\mu_{1} * \mu_{2} * \mu_{3} * \ldots * \mu_{n}$. Then supp $\left(\Phi_{n}\right)$ is uniformly bounded.

Proof. Let $\rho$ be a left-invariant geodesic metric on $\mathcal{G}$. It suffices to show

$$
\rho\left(\operatorname{supp}\left(\Phi_{n}\right)\right) \leq \sum_{i=1}^{n} \rho\left(\operatorname{supp}\left(\mu_{i}\right)\right)
$$

is bounded. Since $\rho$ is $C^{1}$, the sequence $\rho\left(\operatorname{supp}\left(\mu_{i}\right)\right) \rightarrow 0$ exponentially fast. Therefore the infinite series converges.

Lemma 2.2. Let $N \in \mathbb{N}$. Then for $\mathbf{q} \in \mathbb{N}^{d},|\mathbf{q}| \leq N-1$ there exist continuous functions

$$
\mathcal{B}_{\mathbf{q}}: \mathbb{R} \times \mathcal{G} \times \mathcal{L} \times \mathbb{N}^{d} \rightarrow \mathbb{C} \text { and } \mathcal{R}_{\mathbf{q}}: \mathbb{R} \times \mathcal{G} \times \mathcal{L} \times C^{N-|\mathbf{q}|}(\mathcal{G}) \rightarrow \mathbb{C},
$$


such that for any $h \in C^{N}(\mathcal{G})$

$$
\begin{aligned}
\left(D^{\mathbf{q}} h\right)(g \exp (t X))=( & \left.D^{\mathbf{q}} h\right)(g)+\sum_{|\mathbf{n}|=|\mathbf{q}|+1}^{N-1} t^{(|\mathbf{n}|-|\mathbf{q}|)} \mathcal{B}_{\mathbf{q}}(t, g, X, \mathbf{n})\left(D^{\mathbf{n}} h\right)(g) \\
& +\sum_{|\mathbf{n}|=0}^{|\mathbf{q}|} t \mathcal{B}_{\mathbf{q}}(t, g, X, \mathbf{n})\left(D^{\mathbf{n}} h\right)(g) \\
& +t^{(N-|\mathbf{q}|)} \mathcal{R}_{\mathbf{q}}(t, g, X, h) .
\end{aligned}
$$

Furthermore, each $\mathcal{R}_{\mathbf{q}}$ is a linear function of $h$.

Proof. Define $Y:=A d(g) X \in \mathcal{L}$. Then since

$$
\left(D^{\mathbf{q}} h\right)(g \exp (t X))=\left(D^{\mathbf{q}} h\right)(\exp (t Y) g),
$$

and since $D^{\mathbf{q}} h \in \mathbb{C}^{(N-|\mathbf{q}|)}$, the standard Taylor's formula ([22], p. 95) implies there exist continuous functions

$$
r_{\mathbf{q}}: \mathbb{R} \times \mathcal{G} \times \mathcal{L} \times C^{(N-|\mathbf{q}|)}(\mathcal{G}) \rightarrow \mathbb{C}, \text { for } 0 \leq|\mathbf{q}| \leq N-1,
$$

such that $r_{\mathbf{q}}(t, g, X, f)$ is linear with respect to $f$ and

$$
\begin{aligned}
\left(D^{\mathbf{q}} h\right)(g \exp (t X))= & \left(D^{\mathbf{q}} h\right)(g)+\sum_{p=1}^{N-|\mathbf{q}|-1} t^{p} \frac{\left(D(Y)^{p} D^{\mathbf{q}} h\right)(g)}{p !} \\
& +t^{(N-|\mathbf{q}|)} r_{\mathbf{q}}\left(t, g, X, D^{\mathbf{q}} h\right) .
\end{aligned}
$$

Since $D(Y)^{p} D^{\mathbf{q}} \in \mathcal{U}^{p+|\mathbf{q}|}$, the Poincaré-Birkhoff-Witt theorem implies there exist continuous functions

$$
b_{p, \mathbf{q}}: \mathcal{G} \times \mathcal{L} \times \mathbb{N}^{d} \rightarrow \mathbb{R}, \quad \text { for } 1 \leq p \leq N-|\mathbf{q}|-1,
$$

such that

$$
D(Y)^{p} D^{\mathbf{q}}=\sum_{|\mathbf{n}| \leq p+|\mathbf{q}|} b_{p, \mathbf{q}}(g, X, \mathbf{n}) D^{\mathbf{n}}
$$

Therefore

$$
\left(D^{\mathbf{q}} h\right)(g \exp (t X))=\left(D^{\mathbf{q}} h\right)(g)+S+t^{(N-|\mathbf{q}|)} r_{\mathbf{q}}\left(t, g, X, D^{\mathbf{q}} h\right),
$$

where

$$
S=\sum_{p=1}^{N-|\mathbf{q}|-1} t^{p} \sum_{|\mathbf{n}| \leq p+|\mathbf{q}|} \frac{b_{p, \mathbf{q}}(g, X, \mathbf{n})}{p !} D^{\mathbf{n}} .
$$

Decompose $S=S_{1}+S_{2}$ and rearrange the order of summations to obtain

$$
S_{1}=\sum_{|\mathbf{n}|=|\mathbf{q}|+1}^{N-1} t^{(|\mathbf{n}|-|\mathbf{q}|)} \sum_{p=|\mathbf{n}|-|\mathbf{q}|}^{N-|\mathbf{q}|-1} t^{(p+|\mathbf{q}|-|\mathbf{n}|)} \frac{b_{p, \mathbf{q}}(g, X, \mathbf{n})}{p !} D^{\mathbf{n}},
$$

and

$$
S_{2}=\sum_{|\mathbf{n}|=0}^{|\mathbf{q}|} t \sum_{p=1}^{N-|\mathbf{q}|-1} t^{p-1} \frac{b_{p, \mathbf{q}}(g, X, \mathbf{n})}{p !} D^{\mathbf{n}}
$$


We conclude the proof by choosing

$$
\begin{gathered}
\mathcal{B}_{\mathbf{q}}(t, g, X, \mathbf{n})=\sum_{p=|\mathbf{n}|-|\mathbf{q}|}^{N-|\mathbf{q}|-1} t^{(p+|\mathbf{q}|-|\mathbf{n}|)} \frac{b_{p, \mathbf{q}}(g, X, \mathbf{n})}{p !}, \text { for }|\mathbf{n}| \geq|\mathbf{q}|+1 \\
\mathcal{B}_{\mathbf{q}}(t, g, X, \mathbf{n})=\sum_{p=1}^{N-|\mathbf{q}|-1} t^{p-1} \frac{b_{p, \mathbf{q}}(g, X, \mathbf{n})}{p !}, \text { for }|\mathbf{n}| \leq|\mathbf{q}|
\end{gathered}
$$

and

$$
\mathcal{R}_{\mathbf{q}}(t, g, X, h)=r_{\mathbf{q}}\left(t, g, X, D^{\mathbf{q}} h\right)
$$

For any integer $k \in \mathbb{N}$, let $\mathbb{C}^{k}$ denote the $k$-dimensional complex Hilbert space of column vectors $v=\left[v_{1}, \ldots, v_{k}\right]^{T}, v_{i} \in \mathbb{C}$, with vector norm

$$
\|v\|^{2}:=\sum_{i=1}^{m}\left|v_{i}\right|^{2}
$$

and let $\mathbb{C}^{k \times k}$ denote the set of $k \times k$ matrices $A$ having complex entries, with operator norm

$$
\|A\|:=\max _{\|v\|=1}\|A v\| .
$$

Let $\mathcal{L}$ be the Lie algebra of $\mathcal{G}$ and let $d:=\operatorname{dim}(\mathcal{L})$. Fix $N \in \mathbb{N}$. Define

$$
t(n):=\frac{(d+n-1) !}{n !(d-1) !} \text { for } n=0, \ldots, N-1, \text { and } m:=\sum_{n=0}^{N-1} t(n) .
$$

Let $J_{n}$ denote the $t(n)$-dimensional column vector, consisting of the operators $D^{\mathbf{n}}$ (in some arbitrary but fixed order) where $|\mathbf{n}|=n$. Thus $J_{n} h: \mathcal{G} \rightarrow \mathbb{C}^{t(n)}$, for $0 \leq n \leq N-1$. For $h \in \mathbb{C}^{N}(\mathcal{G})$ define the function $\mathcal{J} h: \mathcal{G} \rightarrow \mathbb{C}^{m}$ by

$$
(\mathcal{J} h)(g):=\left[J_{0} h, J_{1} h, \ldots, J_{N-1} h\right]^{T} .
$$

Then $\mathcal{J} h$ depends linearly on $h$ and is called the $(N-1)$-th jet extension of $h$ (with respect to the basis $\left\{X_{i}\right\}$ of $\left.\mathcal{L}\right)$.

The following result extends Lemma 2.2 by describing the effect of right translation on jet extensions.

Lemma 2.3. There exists a continuous matrix-valued function

$$
\mathcal{B}: \mathbb{R} \times \mathcal{G} \times \mathcal{L} \rightarrow \mathbb{C}^{m \times m},
$$

and there exists a continuous vector-valued function

$$
\mathcal{R}: \mathbb{R} \times \mathcal{G} \times \mathcal{L} \times \mathbb{C}^{N}(\mathcal{G}) \rightarrow \mathbb{C}^{m},
$$

such that for $(t, g, X, h) \in \mathbb{R} \times \mathcal{G} \times \mathcal{L} \times \mathbb{C}^{N}(\mathcal{G})$,

$$
(\mathcal{J} h)(g \exp (t X))=(\mathcal{J} h)(g)+t \mathcal{B}(t, g, X)((\mathcal{J} h)(g))+\mathcal{R}(t, g, X, h) .
$$


The matrix valued function $\mathcal{B}$ has the blocked form

$$
\mathcal{B}(t, g, X)=\left(\begin{array}{cccccc}
B_{0,0} & B_{0,1} & t B_{0,2} & \ldots & t^{N-3} B_{0, N-2} & t^{N-2} B_{0, N-1} \\
B_{1,0} & B_{1,1} & B_{1,2} & \ldots & t^{N-4} B_{1, N-2} & t^{N-3} B_{1, N-1} \\
B_{2,0} & B_{2,1} & B_{2,2} & \ldots & t^{N-5} B_{2, N-2} & t^{N-4} B_{2, N-1} \\
\ldots & \ldots & \ldots & \ldots & \ldots & \ldots \\
B_{N-3,0} & B_{N-3,1} & B_{N-3,2} & \ldots & B_{N-3, N-2} & t B_{N-3, N-1} \\
B_{N-2,0} & B_{N-2,1} & B_{N-2,2} & \ldots & B_{N-2, N-2} & B_{N-2, N-1} \\
B_{N-1,0} & B_{N-1,1} & B_{N-1,2} & \ldots & B_{N-1, N-1} & B_{N-1, N-1}
\end{array}\right)
$$

where

$$
B_{i, j}: \mathbb{R} \times \mathcal{G} \times \mathcal{L} \rightarrow \mathbb{C}^{t(i) \times t(j)}, \text { for } 0 \leq i, j \leq N-1,
$$

are continuous matrix-valued functions. The vector-valued function $\mathcal{R}$ has the blocked form

$$
\mathcal{R}(t, g, X, h)=\left(\begin{array}{c}
t^{N} R_{0} \\
t^{N-1} R_{1} \\
t^{N-2} R_{2} \\
\cdots \\
t R_{N-1}
\end{array}\right)
$$

where

$$
R_{i}: \mathbb{R} \times \mathcal{G} \times \mathcal{L} \times \mathbb{C}^{N}(\mathcal{G}) \rightarrow \mathbb{C}^{t(i)} \text { for } 0 \leq i \leq N-1
$$

are continuous vector-valued functions. Furthermore, $R_{i}(t, g, X, h)$ and $\mathcal{R}(t, g, X, h)$ are linear in $h$.

Proof. Follows directly from Lemma 2.2.

The following result describes the effect, on jet extensions, of right-convolution by a refinement mask $\mu$.

Lemma 2.4. There exists a continuous matrix-valued function

$$
\mathcal{A}: \mathcal{G} \times \mathcal{M}(\mathcal{G}) \rightarrow \mathbb{C}^{m \times m},
$$

and a continuous vector-valued function

$$
\mathcal{Q}: \mathcal{G} \times \mathcal{M}(\mathcal{G}) \times \mathbb{C}^{N}(\mathcal{G}) \rightarrow \mathbb{C}^{m},
$$

such that for $(g, \mu, h) \in \mathcal{G} \times \mathcal{M}(\mathcal{G}) \times \mathbb{C}^{N}(\mathcal{G})$,

$$
(\mathcal{J}(h * \mu))(g)=(\mathcal{J} h)(g)+\mathcal{A}(g, \mu)((\mathcal{J} h)(g))+\mathcal{Q}(g, \mu, h) .
$$

The matrix-valued function $\mathcal{A}$ has the blocked form

$$
\mathcal{A}(g, \mu)=\left(\begin{array}{cccccc}
A_{0,0} & A_{0,1} & A_{0,2} & \ldots & A_{0, N-2} & A_{0, N-1} \\
A_{1,0} & A_{1,1} & A_{1,2} & \ldots & A_{1, N-2} & A_{1, N-1} \\
A_{2,0} & A_{2,1} & A_{2,2} & \ldots & A_{2, N-2} & A_{2, N-1} \\
\ldots & \ldots & \ldots & \ldots & \ldots & \ldots \\
A_{N-3,0} & A_{N-3,1} & A_{N-3,2} & \ldots & A_{N-3, N-2} & A_{N-3, N-1} \\
A_{N-2,0} & A_{N-2,1} & A_{N-2,2} & \ldots & A_{N-2, N-2} & A_{N-2, N-1} \\
A_{N-1,0} & A_{N-1,1} & A_{N-1,2} & \ldots & A_{N-1, N-1} & A_{N-1, N-1}
\end{array}\right),
$$


where

$$
A_{i, j}: \mathcal{G} \times \mathcal{M}(\mathcal{G}) \rightarrow \mathbb{C}^{t(i) \times t(j)}, \text { for } 0 \leq i, j \leq N-1,
$$

are continuous. The vector-valued function $\mathcal{Q}$ has the blocked form

$$
\mathcal{Q}(g, \mu, h)=\left(\begin{array}{c}
Q_{0} \\
Q_{1} \\
Q_{2} \\
\cdots \\
Q_{N-1}
\end{array}\right)
$$

where

$$
Q_{i}: \mathcal{G} \times \mathcal{M}(\mathcal{G}) \times \mathbb{C}^{N-i}(\mathcal{G}) \rightarrow \mathbb{C}^{t(i)}, \text { for } 0 \leq i \leq N-1,
$$

are continuous. Furthermore, there exists a left-invariant metric $\rho$ on $\mathcal{G}$, and there exists $c_{1}, c_{2} \in \mathbb{C}^{0}(\mathcal{G})$, such that for any $h \in \mathbb{C}_{c}^{N}(\mathcal{G})$

$$
\left\|A_{i, j}(g, \mu)\right\| \leq c_{1}(g)[\rho(\operatorname{supp}(\mu))]^{\max \{1, j-i\}}
$$

and

$$
\| Q_{i}\left(g, \mu, h\left\|\leq c_{2}(g)[\rho(\operatorname{supp}(\mu))]^{N-i}\right\| h \|_{N-i} .\right.
$$

Furthermore $Q_{i}(g, \mu, h)$ and $\mathcal{Q}(g, \mu, h)$ are linear in $h$.

Proof. Follows directly from Lemma 2.3 .

Definition 2.1. Let $\gamma>0$. A sequence $s_{k}$ of complex numbers is dominated by $\gamma$ if there exists a polynomial $P$ such that

$$
\left|s_{k}\right| \leq P(k) \gamma^{k} .
$$

Clearly any sequence that is dominated by $\gamma<1$ is bounded and is dominated by any $\gamma_{1}>\gamma$. Finite linear combinations of sequences dominated by $\gamma$ are dominated by $\gamma$. Furthermore, if $s_{k}$ is dominated by $\gamma$, then the sequence of partial sums

$$
S_{k}:=\sum_{j=0}^{k} s_{j}
$$

is also dominated by $\gamma$. If sequences $t_{k}, s_{k}$ are dominated by $\gamma_{1}, \gamma_{2}$, respectively, then the sequence $t_{k} s_{k}$ is dominated by $\gamma_{1} \gamma_{2}$.

Henceforth, fix $0<\alpha<1$ and $\beta>1$. Let $\mu_{k}$ be a sequence of refinement masks whose total variations are uniformly bounded $\left|\mu_{k}\right| \leq \beta$ and whose support sequence $\operatorname{supp}\left(\mu_{k}\right)$ converges exponentially fast at rate $\alpha$ to $1 \in \mathcal{G}$. Define convolution products

$$
\Phi_{k}:=\mu_{1} * \mu_{2} * \ldots * \mu_{k} .
$$

We combine Lemma 2.4 with basic properties of dominated sequences to obtain the following powerful result.

Lemma 2.5. For any $N \in \mathbb{N}$ and any $h \in \mathbb{C}_{c}^{N}(\mathcal{G})$, define $h_{k}:=h * \Phi_{k}$. Then for any $0 \leq n \leq N$, and for any $0 \leq i \leq n$, the sequence $\left\|h_{k}\right\|_{i}$ is dominated by $\alpha^{N-n} \beta$. Consequently, $\left\|h_{k}\right\|_{i}$ is dominated by $\alpha^{N-i} \beta$. 
Proof. The second statement is an immediate consequence of the first. We will prove the first statement by backwards induction on $n$. For any continuous compactly supported vector (or scalar) valued function $v$ on $\mathcal{G}$, define

$$
\|v\|_{0}:=\max _{g \in \mathcal{G}}\|v(g)\| .
$$

Since

$$
\left\|h_{k}\right\|_{i} \leq \sum_{j=0}^{i}\left\|J_{j} h_{k}\right\|_{0}
$$

it suffices to show that $i \leq n$ implies $\left\|J_{i} h_{k}\right\|_{0}$ is dominated by $\alpha^{N-n} \beta$. For $n=N$, we have, since the (vector-valued) differential operator $J_{i}$ is right translation invariant and $\mu_{k}$ has total variation less than $\beta$,

$$
\left\|J_{i} h_{k}\right\|_{0}=\left\|\left(J_{i} h_{k-1}\right) * \mu_{k}\right\|_{0} \leq\left\|J_{i} h_{k-1}\right\|_{0}\left|\mu_{k}\right|=\left\|J_{i} h_{k-1}\right\|_{0} \beta .
$$

Therefore $\left\|J_{i} h_{k}\right\|_{0} \leq\left\|J_{i} h\right\|_{0} \beta^{k}$, hence $\left\|J_{i} h_{k}\right\|_{0}$ is dominated by $\beta$. To extend this result for $n<N$ we will use the inequalities in Lemma2.4 We start with $n=N-1$. Since

$$
\left\|J_{i} h_{k}\right\|_{0} \leq\left\|J_{i} k_{k-1}\right\|_{0}+\left\|J_{i} h_{k}-J_{i} h_{k-1}\right\|_{0}
$$

it suffices to show $\left\|J_{i} h_{k}-J_{i} h_{k-1}\right\|_{0}$ is dominated by $\alpha \beta$ for all $0 \leq i \leq N-1$. Define

$$
s_{k}(g):=\left\|\left(J_{i} h_{k}-J_{i} h_{k-1}\right)(g)\right\| .
$$

Lemma 2.4 implies that

$$
s_{k}(g) \leq\left\|Q_{i}\left(g, \mu_{k}, h_{k-1}\right)\right\|+\sum_{j=0}^{N-1}\left\|A_{i, j}\left(g, \mu_{k}\right)\right\|\left\|\left(J_{j} h_{k-1}\right)(g)\right\| .
$$

Lemma 2.1 implies that the supports of $h_{k}$, and therefore the supports of $J_{l} h_{k}$, are uniformly bounded. Therefore (2.1) and (2.2) imply there exists $c>0$ such that

$$
s_{k}(g) \leq c\left[\alpha^{k(N-i)}\left\|h_{k-1}\right\|_{N-i}+\sum_{j=0}^{N-1} \alpha^{k(\max \{1, j-i\})}\left\|\left(J_{j} h_{k-1}\right)\right\|_{0}\right] .
$$

The sequences $\left\|h_{k-1}\right\|_{N-i}$ and $\left\|J_{j} h_{k-1}\right\|_{0}$ are dominated by $\beta$. Since $\alpha<1$, the sequence $\alpha^{k(\max \{1, j-i\})}$, and for $0 \leq i \leq N-1$ the sequence $\alpha^{k(N-i)}$, are dominated by $\alpha$. Therefore the sequence $\left\|J_{i} h_{k}-J_{i} h_{k-1}\right\|_{0}$ is dominated by $\alpha \beta$. This concludes the proof for $n=N-1$. We now assume $n=N-2$ and let $i \leq n$. The first sequence on the right side of (2.1) is clearly dominated by $\alpha^{2} \beta$. It suffices to show the second sequence is dominated by $\alpha^{2} \beta$. For $j \leq i+1$, the sequence $\alpha^{k(\max \{1, j-i\})}$ is dominated by $\alpha$ and since $j \leq N-1$, the sequence $\left\|J_{j} h_{k-1}\right\|$ is dominated by $\alpha \beta$ (since the result was shown for $n=N-1$ ). Therefore the second sequence is dominated by $\alpha^{2} \beta$. For $j \geq i+2$, the sequence $\alpha^{k(\max \{1, j-i\})}$ is dominated by $\alpha^{2}$ and the sequence $\left\|J_{j} h_{k-1}\right\|$ is dominated by $\beta$, hence the second sequence is dominated by $\alpha^{2} \beta$. The proof for $0 \leq n \leq N-3$ proceeds by induction.

Theorem 2.1. There exists $\phi \in \mathcal{E}^{\prime}(\mathcal{G})$ such that $\lim _{k \rightarrow \infty} \Phi_{k}=\phi$. 
Proof. For any $f \in \mathbb{C}^{0}(\mathcal{G})$, define $\left\langle\Phi_{k}, f\right\rangle$ to be the integral of $f$ with respect to the measure $\Phi_{k}$. Since Lemma 2.1 implies that the supports of $\Phi_{k}$ are uniformly bounded, it suffices to show that there exists $N \in \mathbb{N}, c>0$ such that for any $f \in$ $\mathbb{C}_{c}^{N}(\mathcal{G})$, the sequence $\left\langle\Phi_{k}, f\right\rangle$ is a Cauchy sequence whose limit is a linear function of $f$ that is bounded by $c\|f\|_{N}$. This establishes the existence of a compactly supported distribution $\phi \in \mathcal{E}^{\prime}(\mathcal{G})$ having distribution order $\leq N$ such that the linear function has the form $\langle\phi, f\rangle$. Choose $N \geq 1$ such that

$$
\alpha^{N} \beta<1 \text {. }
$$

For any $f \in \mathbb{C}_{c}^{N}(\mathcal{G})$ define an associated function $h \in \mathbb{C}^{N}(\mathcal{G})$ by

$$
h(g):=\Delta(g) f\left(g^{-1}\right)
$$

and define $h_{k}:=h * \Phi_{k}$. A straightforward computation yields

$$
h_{k}(1):=\int h(g) \Phi_{k}\left(g^{-1}\right) d g \int \Delta(g) h\left(g^{-1}\right) \Phi_{k}(g) d g=\left\langle\Phi_{k}, f\right\rangle .
$$

Lemma 2.5 implies that $\left\|h_{k}\right\|_{0}$ is dominated by $\alpha^{N} \beta<1$. Therefore (2.3) implies

$$
\left\|J_{0} h_{k}-J_{0} h_{k-1}\right\| \leq c\left[\left(\alpha^{N} \beta\right)^{k}\|h\|_{N}+\sum_{j=0}^{N-1} \alpha^{k(\max \{1, j\})}\left\|\left(J_{j} h_{k-1}\right)\right\|_{0}\right],
$$

hence the sequence $h_{k}(1)$ is a Cauchy sequence whose limit is a linear function of $h$ that is bounded by $c_{1}\|h\|_{N}$ for some constant $c_{1}>0$. Therefore the limit is a linear function of $f$. Furthermore, since the supports of $\Phi_{k}$ are uniformly bounded and $\Delta$ is analytic, the limit is bounded by $c\|f\|_{N}$ for some constant $c>0$. This completes the proof.

Remark 2.2. Strichartz 53 derived a result, based on the Euclidean Fourier transform, that implies Theorem 2.1 under the assumption that $\mathcal{G}$ is a step 2 stratified nilpotent Lie group.

\section{Dilations}

An automorphism $A$ of a Lie group $\mathcal{G}$ is a dilation if its derivative at $1 \in \mathcal{G}$ is a linear transformation of the Lie algebra $\mathcal{L}$ of $\mathcal{G}$ all of whose eigenvalues have modulus $>1$. We denote the derivative of $A$ by $A$ also and we note that it is a Lie algebra automorphism of $\mathcal{L}$, thus $[A x, A y]=A([x, y])$ where $[\cdot, \cdot]$ denotes the Lie algebra product.

Example 3.1. Parameterize the three-dimensional Heisenberg group $\mathcal{G}$ by $3 \times 3$ upper triangular matrices

$$
\mathcal{G}:=\left\{g(x, y, z)=\left[\begin{array}{ccc}
1 & x & z \\
0 & 1 & y \\
0 & 0 & 1
\end{array}\right]: x, y, z \in \mathbb{R}\right\} .
$$

For any $\lambda_{1}, \lambda_{2}>1$ the function $A$ defined by

$$
A(g(x, y, z)):=g\left(\lambda_{1} x, \lambda_{2} y, \lambda_{1} \lambda_{2} z\right)
$$

is a dilation automorphism having eigenvalues $\lambda_{1}, \lambda_{2}, \lambda_{1} \lambda_{2}$.

Lemma 3.1. If a connected Lie group $\mathcal{G}$ admits a dilation $A$, its Lie algebra $\mathcal{L}$ is nilpotent. Hence $\mathcal{G}$ is a nilpotent Lie group. 


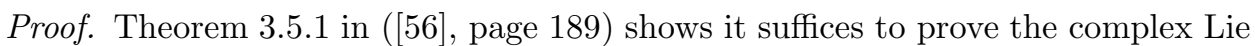
algebra $\mathcal{L} \otimes \mathbb{C}$ (tensor product) is nilpotent. For convenience replace $\mathcal{L}$ by $\mathcal{L} \otimes \mathbb{C}$ and replace $A$ by the induced automorphism on $A$. Clearly the new $A$ has the same eigenvalues as the old $A$, hence it is a dilation. Let $[x, y]$ denote the Lie product of elements $x, y \in \mathcal{L}$, let $\mathcal{L}^{0}=\mathcal{L}$, and for $n>0$, let $\mathcal{L}^{n}=\left[\mathcal{L}, \mathcal{L}^{n-1}\right]$ denote the descending central series for $\mathcal{L}$. By Corollary 3.5.5 in ([56], page 189) it suffices to show there exists an integer $N>0$ such that $\mathcal{L}^{N}=\{0\}$. Assume to the contrary that $\mathcal{L}$ is not nilpotent. Then there exists $N>0$ such that $\left[\mathcal{L}, \mathcal{L}^{N}\right]=\mathcal{L}^{N} \neq\{0\}$. We will show, contrary to our assumption, that $\mathcal{L}^{N}=\{0\}$. We proceed by induction on the dimension of $\mathcal{L}^{N}$. Clearly the central series are invariant under $A$. Let $\lambda \neq 0$ be the eigenvalue of the restriction of $A$ to $\mathcal{L}^{N}$ that has the largest modulus, and let $\mathcal{H}=\mathcal{L}_{\lambda}^{N}$ be the corresponding invariant subspace consisting of $x \in \mathcal{L}^{N}$ such that there exists $M>0$ with $(A-\lambda)^{M} x=0$. It suffices to prove that $[\mathcal{L}, \mathcal{H}]=\{0\}$, since this condition implies that the quotient Lie algebras $\mathcal{H}^{0}=\frac{\mathcal{L}^{0}}{\mathcal{H}}$ and $\mathcal{H}^{N}=\frac{\mathcal{L}^{N}}{H}$ satisfy $\left[\mathcal{H}^{0}, \mathcal{H}^{N}\right]=\mathcal{H}^{N}$ and the dimension of $\mathcal{H}^{N}$ is less than the dimension of $\mathcal{L}^{N}$. The induction hypothesis implies that $\mathcal{H}^{N}=\{0\}$, and consequently $\mathcal{L}^{N}=\mathcal{H}$ and hence $\mathcal{L}^{N}=\left[\mathcal{L}, \mathcal{L}^{N}\right]=[\mathcal{L}, \mathcal{H}]=\{0\}$, thus yielding a contradiction. Therefore it suffices to prove that $[x, y]=0$ whenever $x$ satisfies $(A-\nu)^{p} x=0$ and $(A-\lambda)^{q} y=0$ for some eigenvalue $\nu$ of $A$ and positive integers $p$ and $q$. We proceed by induction on $p+q$. If $p+q=2$, then $A x=\nu x, A y=\lambda y$, thus

$$
A([x, y])=[A x, A y]=\nu \lambda[x, y] .
$$

Therefore $[x, y]=0$ else $[x, y] \in \mathcal{L}^{N}$ is an eigenvector of $A$ whose eigenvalue modulus $|\nu \lambda|>|\lambda|$ (since $A$ is a dilation $|\nu|>1$ ). If $p+q>2$, then either $p>1$ or $q>1$. Assume $p>1$. Then $A x=\nu x+x_{1}$ where $x_{1}$ satisfies $(A-\nu)^{p-1} x_{1}=0$. By the induction assumption $\left[x_{1}, y\right]=0$. Therefore

$$
A([x, y])=[A x, A y]=\lambda\left[\nu x+x_{1}, y\right]=\lambda[\nu x, y]=\nu \lambda[x, y] .
$$

Hence $[x, y]=1$ since $|\nu \lambda|>|\lambda|$. The proof for $q>1$ is similar. This concludes the proof.

Lemma 3.2. The group $\mathcal{G}$ is simply connected.

Proof. Assume to the contrary that $\mathcal{G}$ is not simply connected, let $\mathcal{G}_{s}$ denote the universal covering group of $\mathcal{G}$, let $\rho: \mathcal{G}_{s} \rightarrow \mathcal{G}$ denote the covering homomorphism, and let $\mathcal{K}$ denote the kernel of $\rho$. Since $A$ induces an automorphism on $\mathcal{L}$ it induces an automorphism on $\mathcal{G}_{s}$ that commutes with $\rho$. Therefore $A$ leaves $\mathcal{K}$ invariant. It suffices to prove $\mathcal{K}=\{1\}$. By Lemma 3.1 $\mathcal{L}$ is nilpotent therefore both $\mathcal{G}$ and $\mathcal{G}_{s}$ are nilpotent. By Malcev's theorem [40], exp is an analytic homeomorphism of $\mathcal{L}$ onto $\mathcal{G}_{s}$. Clearly since $\exp (A x)=A(\exp (x))$, for every $g \in \mathcal{G}$ such that $g \neq 1, A^{n} g$ tends to $\infty$ as $n$ increases. Assume $\mathcal{K} \neq\{1\}$. Then $A \mathcal{K} \neq \mathcal{K}$ and there exists $g \in \mathcal{G}_{s}$ such that $g \notin \mathcal{K}$ and $A g \in \mathcal{K}$. Then $\rho(g) \in \mathcal{G}$ and $\rho(g) \neq 1$; however $A \rho(g)=1$. This contradicts the assumption that $A$ is an automorphism of $\mathcal{G}$ and concludes the proof.

Theorem 3.1. If $A$ is an expansive automorphism on $\mathcal{G}$, then the spectral radius $\sigma\left(A^{-1}\right)$ of its inverse is less than 1. Furthermore, for any $\sigma_{0}>\sigma\left(A^{-1}\right)$ there exists a left-invariant metric $\rho$ on $\mathcal{G}$ such that

$$
\rho\left(A^{-1} g, A^{-1} h\right)<\sigma_{0} \rho(g, h), \quad g, h \in \mathcal{G} .
$$


Proof. The first statement is obvious. Given $\sigma_{0}>\sigma\left(A^{-1}\right)$, construct an inner product $(\cdot, \cdot)$ and an associated norm $\|\cdot\|$ on $\mathcal{L}$ such that $\left\|A^{-1} x\right\|<\sigma_{0}\|x\|, x \in \mathcal{L}$. Perform left translation of $(\cdot, \cdot)$ over $\mathcal{G}$ to obtain a left-invariant 1 -form on $\mathcal{G}$ and let $\rho$ denote the corresponding left-invariant geodesic metric on $\mathcal{G}$. The proof is completed by observing that

$$
\rho\left(A^{-1} g, A^{-1} h\right)<\sigma_{0} \rho(g, h), \quad g, h \in \mathcal{G} .
$$

\section{General Refinement Operators}

Let $A$ be a dilation on $\mathcal{G}$ and let $D$ be the corresponding dilation operator. Let $\mu$ be a refinement mask and define the sequence of measures

$$
\mu_{0}:=\mu, \quad \mu_{k}:=D \mu_{k-1}, \quad k=1,2, \ldots .
$$

Then the refinement operator $T=T_{\mu}$ satisfies

$$
T f=D(\mu * f)=\mu_{1} *(D f),
$$

for any $f \in \mathcal{E}(\mathcal{G})$. Iterating this formula leads to

$$
T^{n} f=\mu_{1} * \mu_{2} * \ldots * \mu_{n} *\left(D^{n} f\right) .
$$

Theorem 4.1. For any $f \in \mathcal{E}^{\prime}(\mathcal{G})$ having integral 1 , the cascade sequence $T^{n} f$ converges to $\phi$. Furthermore, $T^{n} h \rightarrow \phi$ for all distributions $h \in \mathcal{D}^{\prime}(\mathcal{G})$ having the form

$$
h=\sum_{\gamma \in \Gamma} b(\gamma) L_{\gamma} f,
$$

where $b \in \ell^{1}(\Gamma)$ and $\sum_{\gamma \in \Gamma} b(\gamma)=1$.

Proof. Follows from Theorem 2.1

Theorem 4.2. There exists a unique minimal compact subset $\mathcal{K} \subset \mathcal{G}$ such that for any open set $\mathcal{U}$ containing $\mathcal{K}$, and for any $f \in \mathcal{E}^{\prime}(\mathcal{G})$, there exists an integer $n(\mathcal{U}, f)$ such that $n \geq n(\mathcal{U}, f)$ implies $\operatorname{supp}\left(T^{n} f\right) \subset \mathcal{U}$.

Proof. By Theorem 3.1 there exists $0<\sigma_{0}<1$ and a left-invariant geodesic metric $\rho$ such that for any compact subset $S \subset \mathcal{G}$,

$$
\rho\left(A^{-1} S\right)<\sigma_{0} \rho(S) .
$$

For $r>0$ define

$$
B(r):=\{g \in \mathcal{G}: \rho(g, 1) \leq r\} .
$$

Clearly if $f \in \mathcal{E}^{\prime}(\mathcal{G})$ and $\operatorname{supp}(f) \subset B(r)$, then $\operatorname{supp}(T f) \subset B\left(\sigma_{0}(\rho(\operatorname{supp}(\mu))+r)\right)$. Therefore $B(\rho, r) \subset B(\rho, r)$ whenever

$$
r \geq \frac{\sigma_{0} \rho(\operatorname{supp}(\mu))}{1-\sigma_{0}} .
$$

This establishes existence of a compact set $\mathcal{K}$ having the asserted property. Uniqueness and minimality are obtained by forming the intersections of all such compact sets. 


\section{Discrete Refinement Operators}

Let $\mathcal{G}$ be a connected Lie group that admits a dilation automorphism $A$. Then $\mathcal{G}$ must be simply connected and nilpotent. Also assume there exists a uniform subgroup $\Gamma \subset \mathcal{G}$. This means $\Gamma$ is discrete and has compact quotient. A necessary and sufficient condition for there to exist a uniform subgroup of $\mathcal{G}$ is that $\mathcal{L}$ has a rational structure and $\log (\Gamma)$ generates, over the rationals $Q$, a dense rational subspace $\mathcal{L}_{Q} \subset \mathcal{L}$, see $([8]$, page 200, Theorem 5.1.8). Furthermore, assume that $A \Gamma \subset \Gamma$. Let $\Gamma_{o}$ contain 1 and be a complete set of representatives of the right cosets of $A \Gamma$ in $\Gamma$, and let $F$ be a fundamental domain for the right cosets of $\Gamma$ in $\mathcal{G}$. Then

$$
(A \Gamma) \Gamma_{o}=\Gamma
$$

and

$$
\Gamma F=\mathcal{G} .
$$

Let meas $(S)$ denote the Haar measure of a subset $S \subset \mathcal{G}$ and let $\operatorname{card}(R)$ denote the cardinality of a discrete set $R$. Clearly $\operatorname{card}\left(\Gamma_{o}\right)=\operatorname{card}((A \Gamma) \Gamma)$.

Lemma 5.1. The complete set of representatives $\Gamma_{o}$ has $\operatorname{card}\left(\Gamma_{o}\right)=|A|$ elements.

Proof. Since $\Gamma_{o} F$ equals the union of $\operatorname{card}\left(\Gamma_{o}\right)$ left-translates of $F$ and the pairwise intersections of these translates have measure 0

$$
\operatorname{meas}\left(\Gamma_{o} F\right)=\operatorname{card}\left(\Gamma_{o}\right) \text { meas }(F) .
$$

Substituting (5.1) into (5.2) gives $(A \Gamma)\left(\Gamma_{o} F\right)=\mathcal{G}$. Therefore $\Gamma_{o} F$ is a fundamental domain for $A \Gamma$. However, since $(A \Gamma)(A F)=A \mathcal{G}=\mathcal{G}$, it follows that $A F$ is also a fundamental domain for $A \Gamma$. Therefore,

$$
\operatorname{meas}\left(\Gamma_{o} F\right)=\operatorname{meas}(A F)=|A| \text { meas }(F) .
$$

The proof follows from (5.3) and (5.4).

Define the tile $\mathcal{T} \subset \mathcal{G}$ by

$$
\mathcal{T}:=\left(A^{-1} \Gamma_{o}\right)\left(A^{-2} \Gamma_{o}\right)\left(A^{-3} \Gamma_{o}\right) \cdots .
$$

Since $A$ is a dilation, $\mathcal{T}$ is compact and $A \mathcal{T}=\Gamma_{o} \mathcal{T}$. Furthermore the pairwise intersections of the $|A|$ translates of $\mathcal{T}$ by elements in $\Gamma_{o}$ have measure 0 since the total measure of $A \mathcal{T}$ equals $|A|$ times the measure of $\mathcal{T}$.

Lemma 5.2. The tile $\mathcal{T}$ has positive measure. Furthermore, the characteristic function of $\mathcal{T}$ is $T_{c}$-refinable, where $c$ is the characteristic function of $\Gamma_{o}$.

Proof. Define $G_{o}:=\Gamma \mathcal{T}$. It suffices to prove $G_{o}=\mathcal{G}$, since this would imply that meas $(\mathcal{T}) \geq \operatorname{meas}(F)$ Since $G_{o}=\Gamma \mathcal{T}=(A \Gamma) \Gamma_{o} \mathcal{T}=(A \Gamma)(A \mathcal{T})$, it follows that $A^{-1} G_{o}=\Gamma \mathcal{T}=G_{o}$. Now $1 \in \Gamma_{o}$ implies that $1 \in \mathcal{T}$ thus $\Gamma \subset G_{o}$. Therefore $A^{-j} \Gamma \subset G_{o}$, for $j=0,1, \ldots$, hence $\Gamma \cup A^{-1} \Gamma \cup A^{-2} \Gamma \cdots \subset G_{o}$. Therefore $G_{o}$ is dense in $\mathcal{G}$. Since $G_{o}$ is closed $G_{O}=\mathcal{G}$. Let $\phi$ be the characteristic function of $\mathcal{T}$ and let $c$ be the characteristic function of $\Gamma_{o}$. Clearly $\Gamma_{o} \mathcal{T}=A \mathcal{T}$ implies that $\phi$ is $T_{c}$ refinable.

Theorem 5.1. Let $f$ be a Schoenberg distribution, let $c$ be a fundamental refinement mask, and let $T=T_{c}$ be the $(A, c)$-refinement operator. Then $T f$ is a Schoenberg distribution. 
Proof. It suffices to show $S:=\sum_{p \in \Gamma} L_{p} T \phi=1$. Direct computation yields

$$
\begin{aligned}
S & =\sum_{p \in \Gamma} \sum_{\gamma \in \Gamma} \sum_{r \in \Gamma_{o}}|A| c((A \gamma) r) L_{p} D L_{A \gamma} L_{r} \phi \\
& =\sum_{p \in \Gamma} \sum_{\gamma \in \Gamma} \sum_{r \in \Gamma_{o}}|A| c((A \gamma) r) L_{p} L_{\gamma} D L_{r} \phi \\
& =\sum_{q \in \Gamma} \sum_{\gamma \in \Gamma} \sum_{r \in \Gamma_{o}}|A| c((A \gamma) r) L_{q} D L_{r} \phi \\
& =\sum_{q \in \Gamma} \sum_{r \in \Gamma_{o}} L_{q} D L_{r} \phi \\
& =D \sum_{q \in \Gamma} \sum_{r \in \Gamma_{o}} L_{A q} L_{r} \phi \\
& =1 .
\end{aligned}
$$

Corollary 5.1. If $c$ is fundamental and $\phi$ is an $(A, c)$-refinable distribution with integral 1 , then $\phi$ is a Schoenberg distribution.

Proof. Let $\chi_{F}$ denote the characteristic function of the fundamental domain $F$ of $\Gamma$, and construct $f=\alpha \chi_{F}$ where $\alpha>0$ is chosen so $\int f=1$. Theorem 5.1]implies $T^{n} f$ is a sequence of Schoenberg distributions and Theorem 4.1] implies $T^{n} f$ converges to $\phi$. Clearly $\phi$ is a Schoenberg distribution since for any test function $h \in \mathcal{D}(\mathcal{G})$

$$
\left\langle\sum_{\gamma \in \Gamma} L_{\gamma} \phi, h\right\rangle=\lim _{n \rightarrow \infty}\left\langle\sum_{\gamma \in \Gamma} L_{\gamma} T^{n} f, h\right\rangle=\langle 1, h\rangle .
$$

\section{Transition Operators}

Let $A, \Gamma$ be as in $\$ 5$ and let $c$ be a refinement mask. Define the transition operator $W$ and the subdivision operator $S$ as in $\$ 2$ Then $W$ can be expressed as

$$
(W b)(\gamma)=\sum_{p \in \Gamma} \sum_{q \in \Gamma}|A| c(p) \overline{c(q)} b\left(p^{-1}(A \gamma) q\right) .
$$

If the group $\mathcal{G}$ is abelian, $\mathcal{G}=\mathbb{R}^{d}$ and the operators can be expressed using the autocorrelation $c_{a u}=c * \tilde{c}$ where $\tilde{c}(g):=\overline{c\left(g^{-1}\right)}$ as

$$
(W b)(\gamma)=\sum_{\alpha \in \Gamma}|A| c_{a u}(A \gamma-\alpha) b(\alpha),
$$

and

$$
(S b)(\alpha)=\sum_{\gamma \in \Gamma}|A| c_{a u}(\alpha-A \gamma) b(\gamma) .
$$

Lemma 6.1. For any $f \in \ell^{1}(\Gamma)$,

$$
\langle S 1, f\rangle=|A| \sum_{r \in \Gamma_{o}} \sum_{s \in \Gamma_{o}} c_{r} \overline{c_{s}} \sum_{\nu \in \Gamma} f\left(r^{-1}(A \nu) s\right) .
$$


Proof. Clearly

$$
\begin{aligned}
\langle S 1, f\rangle & =\langle 1, W f\rangle=\sum_{\gamma \in \Gamma}(W f)(\gamma) \\
& =\sum_{\gamma \in \Gamma} \sum_{p \in \Gamma} \sum_{q \in \Gamma}|A| c(p) \overline{c(q)} f\left(p^{-1}(A \gamma) q\right) .
\end{aligned}
$$

Use the fact $(A \Gamma) \Gamma_{o}=\Gamma$ to obtain

$$
\langle S 1, f\rangle=\sum_{\gamma \in \Gamma} \sum_{p \in \Gamma} \sum_{r \in \Gamma_{o}} \sum_{q \in \Gamma} \sum_{s \in \Gamma_{o}}|A| c(A(p) r) \overline{c(A(q) s)} f\left(r^{-1} A\left(p^{-1} \gamma q\right) s\right) .
$$

Substitute $\nu=p^{-1} \gamma q$ and use the fact that summing over $\gamma \in \Gamma$ is equivalent to summing over $\nu \in \Gamma$ to obtain the result.

Theorem 6.1. The refinement mask $c$ is fundamental if and only if the constant function $1 \in \mathbb{C}(\Gamma)$ is an eigenvector of $S$ with eigenvalue 1 .

Proof. For the only if part assume $c$ is fundamental. Then substitute $c_{r}=c_{s}=$ $|A|^{-1}$ in (6.1) to obtain

$$
\begin{aligned}
\langle S 1, f\rangle & =|A|^{-1} \sum_{r, s \in \Gamma_{o}} \sum_{\nu \in \Gamma} f\left(r^{-1}(A \nu) s\right) \\
& =|A|^{-1} \sum_{r \in \Gamma_{o}} \sum_{\gamma \in \Gamma} f(\gamma)=\sum_{\gamma \in \Gamma} f(\gamma)
\end{aligned}
$$

Since this is valid for every $f \in \ell^{1}(\Gamma), S 1=1$. For the if part assume $S 1=1$ and let $f=\delta_{1}$ in 6.1 to obtain

$$
1=|A| \sum_{r \in \Gamma_{o}} \sum_{s \in \Gamma_{o}} c_{r} \overline{c_{s}} \sum_{\nu \in \Gamma} \delta_{1}\left(r^{-1}(A \nu) s\right)=|A| \sum_{r \in \Gamma_{o}}\left|c_{r}\right|^{2} .
$$

Since $c$ is a refinement mask,

$$
\sum_{\gamma \in g G} c(\gamma)=\sum_{r \in g G_{o}} c_{r}=1
$$

Combining these two equations shows $c_{r}=|A|^{-1}, r \in \Gamma$, therefore $c$ is fundamental.

Theorem 6.2. The refinement and transition operators are related by

$$
[T \phi, T \psi]=W([\phi, \psi])
$$


Proof. For any $\gamma \in \Gamma$,

$$
\begin{aligned}
{[T \phi, T \psi](\gamma) } & =(T \phi) *(\widetilde{T \psi})(\gamma) \\
& =\int_{g \in \mathcal{G}}(T \phi)(g)(\widetilde{T \psi})\left(g^{-1} \gamma\right) d g \\
& =\int_{g \in \mathcal{G}}(T \phi)(g) \overline{(T \psi)\left(\gamma^{-1} g\right)} d g \\
& =|A|^{2} \sum_{p \in \Gamma} \sum_{q \in \Gamma} c(p) \overline{c(q)} \int_{g \in \mathcal{G}}\left(L_{p} \phi\right)(A g) \overline{\left(L_{q} \psi\right)\left(A\left(\gamma^{-1} g\right)\right)} d g \\
& =|A| \sum_{p \in \Gamma} \sum_{q \in \Gamma} c(p) \overline{c(q)} \int_{g \in \mathcal{G}} \phi\left(p^{-1} g\right) \overline{\psi\left(q^{-1}\left(A \gamma^{-1}\right) g\right)} d g \\
& =|A| \sum_{p \in \Gamma} \sum_{q \in \Gamma} c(p) \overline{c(q)} \int_{g \in \mathcal{G}} \phi(g) \overline{\psi\left(q^{-1}\left(A \gamma^{-1}\right) p g\right)} d g \\
& =|A| \sum_{p \in \Gamma} \sum_{q \in \Gamma} c(p) \overline{c(q)}[\phi, \psi]\left(p^{-1}(A \gamma) q\right) \\
& =(W([\phi, \psi]))(\gamma) .
\end{aligned}
$$

Remark 6.1. (6.2) means the operator $[\cdot, \cdot]$ is an intertwining operator for $W$, or equivalently, that the following diagram commutes

$$
\begin{array}{ccc}
L^{2}(\mathcal{G}) \times L^{2}(\mathcal{G}) & \stackrel{T \times T}{\longrightarrow} & L^{2}(\mathcal{G}) \times L^{2}(\mathcal{G}) \\
\downarrow[\cdot, \cdot] & & \downarrow[\cdot, \cdot] \\
\mathbb{C}(\Gamma) & \stackrel{W}{\longrightarrow} & \mathbb{C}(\Gamma) .
\end{array}
$$

Let $\mathcal{K} \subset \mathcal{G}$ be the compact subset in Theorem 4.2 and define the finite subset $\Omega \subset \Gamma$ by

$$
\Omega:=\mathcal{K K}^{-1} \cap \Gamma .
$$

Theorem 6.3. For every finitely supported $b \in \mathbb{C}(\Gamma)$, there exists an integer $n(b)$ such that $n \geq n(b)$ implies supp $\left(W^{n} b\right) \subset \Omega$. Furthermore, $\Omega$ is the minimal set with this property.

Proof. Let $\mathcal{U}$ be an open subset containing $\mathcal{K}$ such that the open subset $\mathcal{O}:=\mathcal{U U}^{-1}$ satisfies

$$
\Omega=\mathcal{O} \cap \Gamma \text {. }
$$

Let $\mathcal{T}$ be a tile constructed from $\Gamma_{o}$ as in Lemma [5.2, construct $\phi:=$ characteristic function of $\mathcal{T}$ and $\psi:=\sum_{\gamma \in \Gamma_{o}} \bar{b}(\gamma) L_{\gamma^{-1}} \phi$. Clearly $[\phi, \psi]=b$. Theorem 4.2 implies there exists $n(b)>0$ such that $n \geq n(b) \operatorname{implies} \operatorname{supp}\left(T^{n} \phi\right) \subset \mathcal{U}$, and $\operatorname{supp}\left(T^{n} \psi\right) \subset \mathcal{U}$. Therefore, (6.2) and (6.3) imply that for $n \geq n(b)$,

$$
\operatorname{supp}\left(W^{n} b\right)=\operatorname{supp}\left(\left[T^{n} \phi, T^{n} \psi\right]\right) \subset \Omega,
$$

and concludes the proof. 
Define the restricted transition operator $W_{r}$ to be the restriction of $W$ to functions supported on $\Omega$. The operator $W_{r}$ satisfies condition $E$ if it has a simple eigenvalue 1 and all its other eigenvalues lie inside the unit circle. The operator $W_{r}$ satisfies the extended condition $E$ if all of its eigenvalues lie inside or on the unit circle and all the eigenvalues on the unit circle are nondegenerate (their geometric multiplicities are equal to their algebraic multiplicities).

Theorem 6.4. If $W_{r}$ satisfies extended condition $E$, then the compactly supported refinable distribution $\phi$ is in $L^{2}(\mathcal{G})$. Furthermore, for every $f \in L^{2}(\mathcal{G})$ having compact support and integral $1, T^{n} f$ converges weakly in $L^{2}(\mathcal{G})$ to $\phi$.

Proof. Let $f \in L^{2}(\mathcal{G})$ have compact support. Theorem 4.1 implies that

$$
T^{n} f \rightarrow \phi
$$

in the space of distributions $\mathcal{E}^{\prime}(\mathcal{G})$. Theorem 6.2 implies that

$$
\left\|T^{n} f\right\|_{2}^{2}=\left[T^{n} f, T^{n} f\right](1)=\left(W^{n}([f, f])\right)(1) .
$$

Since $[f, f]$ has finite support, Theorem 6.3 implies there exists $N>0$ such that

$$
\text { support } W^{n}([f, f]) \subset \Omega, \quad n \geq N \text {. }
$$

Therefore

$$
W^{k} W^{N}([f, f])=W_{r}^{k} W^{N}([f, f]), \quad k \geq 1 .
$$

Therefore, if $W_{r}$ satisfies restricted condition E, then the sequence $W^{n}([f, f])(1)$ is bounded and it must have a subsequence that converges weakly to a function $h \in L^{2}(\mathcal{G})$. Since the topology of weak convergence in $L^{2}(\mathcal{G})$ is stronger (finer) than the topology of $\mathcal{E}^{\prime}(\mathcal{G})$, it follows that $h=\phi$. Furthermore, $T^{n} f$ converges weakly to $\phi$, otherwise it has a subsequence which converges to another function in $L^{2}(\mathcal{G})$ which is impossible. This completes the proof.

Theorem 6.5. The cascade sequence $T^{n} f$ converges strongly in $L^{2}(\mathcal{G})$ for any Schoenberg function $f \in L^{2}(\mathcal{G})$ if and only if the restricted transition operator $W_{r}$ satisfies condition $E$ and the refinement mask $c$ is fundamental.

Proof. Assume $W_{r}$ satisfies condition E and $c$ is fundamental. Let $f$ be a Schoenberg function in $L^{2}(\mathcal{G})$. Then by Theorem 6.4, the cascade sequence $T^{n} f$ converges weakly to a refinable $\phi \in L^{2}(\mathcal{G})$. It suffices to prove $\left\|T^{n} f\right\|_{2} \rightarrow\|\phi\|_{2}$, as $n \rightarrow \infty$.

Theorem 5.1 and Corollary 5.1 imply

$$
\sum_{\gamma \in \Gamma} L_{\gamma}\left(T^{n} f\right)=1
$$

and

$$
\sum_{\gamma \in \Gamma} L_{\gamma}(\phi)=1
$$

Conjugating both sides of (6.4), multiplying by $T^{n} f$, integrating over $\mathcal{G}$, and using the fact that the integral of $T^{n} f$ is 1 , yields

$$
\sum_{\gamma \in \Gamma}\left[T^{n} f, T^{n} f\right](\gamma)=1 .
$$


Similarly, (6.5) leads to

$$
\sum_{\gamma \in \Gamma}[\phi, \phi](\gamma)=1
$$

Theorem 5.1 and condition E imply there exists $\beta \in \mathbb{R}$ such that

$$
\lim _{n \rightarrow \infty}\left[T^{n} f, T^{n} f\right]=\lim _{n \rightarrow \infty} W_{r}^{n}([f, f])=\beta[\phi, \phi] .
$$

The two previous relations imply that $\beta=1$, and the result follows.

Conversely, assume that

$$
\lim _{n \rightarrow \infty}\left\|T^{n} f-\phi\right\|_{2} \rightarrow 0,
$$

for all Schoenberg functions $f \in L^{2}(\mathcal{G})$. Let $f$ be the indicator function of a fundamental domain $F$ for $\Gamma$. For each $\alpha \in \Gamma$, define the sequence of functions $b_{n}^{\alpha}$, on $\Gamma$ by

$$
b_{n}^{\alpha}:=\left[T^{n} f, T^{n}\left(L_{\alpha^{-1}} f\right)\right]=W^{n}\left(\left[f, L_{\alpha^{-1}} f\right]\right), \quad n \in \mathbb{N} .
$$

Clearly

$$
b_{0}^{\alpha}=\left[f, L_{\alpha}^{-1} f\right]=\delta_{\alpha} .
$$

Therefore,

$$
b_{n}^{\alpha}=W^{n} \delta_{\alpha},
$$

where $\delta_{\alpha}(\alpha)=1$ and $\delta_{\alpha}(x)=0$ for $x \neq \alpha$. Since each $L_{\alpha^{-1}} f$ is a Schoenberg function in $L^{2}(\mathcal{G})$, the strong convergence assumption implies

$$
\lim _{n \rightarrow \infty} b_{n}^{\alpha}=[\phi, \phi] .
$$

Therefore,

$$
\lim _{n \rightarrow \infty} W^{n} \delta_{\alpha}=[\phi, \phi] .
$$

This clearly implies that for all finitely supported functions $b$ on $\Gamma$,

$$
\lim _{n \rightarrow \infty} W^{n} b=\beta[\phi, \phi],
$$

where

$$
\beta=\sum_{\gamma \in \Gamma} b(\gamma) .
$$

Therefore the matrix sequence $W_{r}^{n}$ converges and all eigenvalues of $W_{r}$ are either inside the unit circle or equal to 1 . Furthermore 1 is a simple eigenvalue of $W_{r}$ since $[\phi, \phi]$ is the unique eigenvector corresponding to the eigenvalue 1 . This shows $W_{r}$ satisfies condition $\mathrm{E}$. We now show that $c$ is fundamental. Let $v$ be an eigenvector of the adjoint $W_{r}^{*}$. By Theorem [6.1, it suffices to prove $v$ is a constant function on $\Gamma$. For $n \in \mathbb{N}$,

$$
v(\alpha)=\sum_{\gamma \in \Gamma} v(\gamma) \delta_{\alpha}(\gamma)=\sum_{\gamma \in \Gamma}\left(\left(W_{r}^{n}\right)^{*} v\right)(\gamma) \delta_{\alpha}(\gamma)=\sum_{\gamma \in \Gamma} v(\gamma)\left(W_{r}^{n} \delta_{\alpha}\right)(\gamma)
$$

Let $n \rightarrow \infty$, and use the fact

$$
\lim _{n \rightarrow \infty} W_{r}^{n} \delta_{\alpha}=[\phi, \phi]
$$


to obtain

$$
v(\alpha)=\left(\sum_{\gamma \in \Gamma} v(\gamma)\right)[\phi, \phi]
$$

This completes the proof.

A function $f \in L^{2}(\mathcal{G})$ is stable if the translates $\Gamma f \subset L^{2}(\mathcal{G})$ form a Riesz basis for the closed linear subspace $\operatorname{span}(\Gamma f)$ of $L^{2}(\mathcal{G})$ that it generates. This means that the mapping

$$
\mathcal{B}: \ell^{2}(\Gamma) \rightarrow \operatorname{span}(\Gamma f)
$$

defined by

$$
\mathcal{B}(a):=\sum_{\gamma \in \Gamma} a(\gamma) L_{\gamma} f
$$

is a bounded injective mapping with bounded inverse. Clearly if $f \in L^{2}(\mathcal{G})$ is stable, then the operator

$$
\mathcal{B}^{*} \mathcal{B}: \ell^{2}(\Gamma) \rightarrow \ell^{2}(\Gamma)
$$

is the convolution operator

$$
\mathcal{B}^{*} \mathcal{B} a=[f, f] * a
$$

Furthermore,

\section{$\mathcal{B}^{*} \mathcal{B}$}

is bounded, one-to-one, onto, and has a bounded inverse that is also a convolution operator. Therefore there exists a function, which we denote by $[[f, f]] \in \mathbb{C}(\Gamma)$, such that

$$
[[f, f]] *[f, f]=\delta_{1} .
$$

Bounded translational invariant linear operators on $\ell(\Gamma)$ are called multipliers. If $\Gamma$ is abelian, then multipliers coincide with convolution with functions whose Fourier transforms are in $L^{\infty}(\widehat{\Gamma})$, where $\widehat{\Gamma}$ is the compact abelian dual group of $\Gamma$.

Theorem 6.6. If for any $f \in L^{2}(\mathcal{G})$ having compact support, the cascade algorithm $T^{n} f$ converges weakly in $L^{2}(\mathcal{G})$ to a multiple of $\phi \in L^{2}(\mathcal{G})$ and $\phi$ is stable and $[[\phi, \phi]] \in \ell^{1}(\Gamma)$, then $W_{r}$ satisfies condition $E$.

Proof. Let $a \in \mathbb{C}(\Gamma)$ be an eigenvector of $W_{r}$, with eigenvalue $\lambda$, whose support is contained in $\Omega$. Define

$$
b:=a *[[\phi, \phi]]
$$

and define $h \in L^{2}(\mathcal{G})$ by

$$
h:=\sum_{\gamma \in \Gamma} b(\gamma) L_{\gamma} \phi .
$$

Therefore $[h, \phi]=a$. Since $[[\phi, \phi]] \in \ell^{1}(\Gamma)$, there exists $\alpha \in \mathbb{C}$ such that

$$
\lim _{n \rightarrow \infty} T^{n} h=\alpha \phi
$$

where the convergence is in the weak sense. Therefore, since $\phi=T^{n} \phi$,

$$
\lim _{n \rightarrow \infty}\left[T^{n} h, T^{n} \phi\right]=\alpha[\phi, \phi]
$$


Theorem 6.2 implies that

$$
\lim _{n \rightarrow \infty} \lambda^{n} a=\alpha[\phi, \phi] .
$$

Therefore $|\lambda|<1$, or $\lambda=1$ and 1 is a simple eigenvalue. Thus condition $\mathrm{E}$ holds and the proof is complete.

\section{REFERENCES}

1. L. Bagget, A. Carey, W. Moran and P. Ohring, General existence theorems for orthonormal wavelets, an abstract approach, publications of the Research Institute of Mathematical Sciences, Kyoto University, \#1, 31 (1995), 95-111. MR 96c:42060

2. G. Birkhoff, A note on topological groups, Compositio Mathematica, 3 (1936), 427-430.

3. N. Bourbaki, Groupes et algèbres de Lie. Chapitre I. Elements de Mathematique. Fasciule XXVI, Hermann, Paris, 1971. MR 42:6159

4. A. S. Cavaretta, W. Dahmen and C. A. Micchelli, Stationary subdivision, Memoirs of the American Mathematical Society, 93 (1991), 1-186. MR 92h:65017

5. A. Cohen, Ondelettes, analyses multiresolutions et traitement numerique du signal, $\mathrm{PhD}$ thesis, Universite Paris IX, Dauphine, 1990.

6. A. Cohen and I. Daubechies, A stability criterion for biorthogonal wavelet bases and their subband coding scheme, Duke Mathematical Journal, 68 (1992), 313-335. MR 94b:94005

7. A. Cohen, I. Daubechies and J. C. Feauveau, Biorthogonal basis of compactly supported wavelets, Communications on Pure and Applied Mathematics, 45 (1992), 485-560. MR 93e:42044

8. L. Cornwin and F. P. Greenleaf, F. P., Representations of Nilpotent Lie Groups and their Applications, Cambridge University Press, 1990. MR 92b:22007

9. X. Dai and D. R. Larson, Wandering vectors for unitary systems and orthonormal wavelets, to appear in Memoirs of the American Mathematical Society. MR 98m:47067

10. I. Daubechies, Orthonormal bases of compactly supported wavelets, Communications on Pure and Applied Mathematics, 41 (1988), 909-996. MR 90m:42039

11. I. Daubechies, Ten lectures on wavelets, CBMF Conference Series in Applied Mathematics, 61, SIAM, Philadephia, 1992. MR 93e:42045

12. G. Deslauriers and S. Dubuc, Interpolation dyadique, in Fractals, Dimensions Non Entiérs et Applications (edited by G. Cherbit), Masson, Paris, 1987, pp. 44-45.

13. J. Dixmier and W. G. Lister, Derivations of nilpotent Lie algebras, Proceedings of the American Mathematical Society, 8 (1957), 155-158. MR 18:659a

14. J. L. Dyer, A nilpotent Lie algebra with nilpotent automorphism group, Bulletin of the American Mathematical Society, 76 (1970), 52-56. MR 40:2789

15. N. Dyn, J. A. Gregory and D. Levin, Analysis of uniform binary subdivision schemes for curve design, Constructive Approximation, 7 (1991), 127-147. MR 92d:65027

16. T. Eirola, Sobolev characterization of solutions of dilation equations, SIAM Journal of Mathematical Analysis, 23 (1992), 1015-1030. MR 93f:42056

17. G. B. Folland, Subelliptic estimates and function spaces on nilpotent Lie groups, Arkiv fur Mathematik, 13 (1975), 161-207. MR 58:13215

18. G. B. Folland, Harmonic Analysis in Phase Space, Princeton University Press, Princeton, New Jersey, 1989.

19. S. A. Gall, Linear Analysis and Representation Theory, Springer-Verlag, New York, 1973.

20. A. Grothendieck, Produits tensoriels et espaces nucléaires, Memoirs of the American Mathmatical Society, 16 (1955).

21. A. Haar, Zur Theorie der orthogonalen Funktionensysteme, Mathematische Annalen, 69 (1910), 331-371.

22. S. Helgason, Differential Geometry and Symmetric Spaces, Academic Press, New York, 1962. MR 26:2986

23. P. N. Heller and R. O. Wells, Jr., The spectral theory of multiresolution operators and applications, in Wavelets: Theory, Algorithms, and Applications, (edited by C. K. Chui, L. Montefusco, L. Puccio), Academic Press, 1994, pp. 13-31. MR 96a:42046

24. L. Hervé, Construction et regularite des fonctions d' echelle, SIAM J. Math. Anal., 5 (1995), 26.

25. R. Hermann, Lie Groups for Physicists, Benjamin, New York, 1966. MR 35:4327 
26. J. E. Humphreys, Introduction to Lie Algebras and Representation Theory, Springer-Verlag, New York, 1972. MR 48:2197

27. N. Jacobson, A note on automorphisms and derivations of Lie algebras, Proceedings of the American Mathematical Society, 6 (1995), 281-283. MR 16:897e]

28. N. Jacobson, Lie Algebras, Wiley Interscience, New York, 1962. MR 26:1345

29. R. Q. Jia, Subdivision Schemes in $L_{p}$ space, Advances in Computational Mathematics, 3 (1995), 309-341. MR 96d:65028

30. R. Q. Jia and Z. Shen, Multiresolution and wavelets, Procedings Edinburgh Mathematical Society, 37 (1994), 271-300. MR 95h:42035

31. S. Kakutani, Über die Metrization der Topologischen Gruppen, Proceedings of the Imperial Academay of Tokyo, 12 (1936), 82-84.

32. W. Lawton, Tight frames of compactly supported affine wavelets, Journal of Mathematical Physics, \#8, 31 (1990), 1898-1901. MR 92a:81068

33. W. Lawton, Necessary and sufficient conditions for constructing orthonormal wavelet bases, Journal of Mathematical Physics, \#1, 32 (1991), 57-61. MR 91m:81100

34. W. Lawton, Multiresolution properties of the wavelet-Galerkin operator, Journal of Mathematical Physics, \#6, 32 (1991), 1440-1443. MR 92f:42038

35. W. Lawton and H. Resnikoff, Multidimensional Wavelet Bases, Technical Report, AWARE, Inc., Bedford, Massachusettes, 1991.

36. W. Lawton, S. L. Lee and Z. Shen, Stability and orthonormality of multivariate refinable functions, SIAM Journal of Mathematical Analysis, \#4, 28 (1997), 999-1014. MR 98d:41027

37. W. Lawton, S. L. Lee and Z. Shen, Convergence of multidimensional cascade algorithm, Numerische Mathematik, 78 (1998), 427-438. MR 98k:41027

38. P. G. Lemarie, Base d'ondelettes sur les groups de Lie stratifies, Bulletin Society Math. France, 117 (1989), 211-232. MR 90j:42066

39. R. Long and D. Chen, Biorthogonal wavelet bases on $\mathbb{R}^{d}$, Appl. Comp. Harmonic Anal., 2 (1995), 230-242. MR 96j:42012

40. A. Malcev, On a class of homogeneous spaces, Izvestia Akademia Nauk SSSR Ser. Mat., 13 (1942), 9-32, American Mathematical Society Translations, vol. 39, 1949. MR 10:507d

41. K. Maurin, General Eigenfunction Expansions and Unitary Representations of Topological Groups, Polish Scientific Publishers, Warsaw, 1968. MR 40:645

42. Y. Meyer, Wavelets and Operators, Cambridge University Press, Cambridge, 1992. MR 94f:42001

43. P. J. Olver, Applications of Lie Groups to Differential Equations, Springer-Verlag, New York, 1993. MR 94g:58260

44. W. Rudin, Functional Analysis, McGraw Hill, New York, 1973. MR 51:1315

45. W. Rudin, Real and Complex Analysis, McGraw Hill, New York, 1974. MR 49:8783

46. H. Samelson, Notes on Lie Algebras, Springer-Verlag, New York, 1990. MR 91h:17006

47. O. L. Sattinger and O. L. Weaver, Lie Groups and Algebras with Applications to Physics, Geometry, and Mechanics, Springer-Verlag, New York, 1986.

48. Z. Shen, Refinable function vectors, SIAM Journal of Mathematical Analysis, 29 (1998), 235250. MR 99d:41038

49. I. J. Schoenberg, Contributions to the problem of approximation of equidistant data by analytic functions, Quarterly Applied Mathematics., 4 (1946), 112-141. MR 8:55d

50. L. Schwartz, Theorie des distributions, Hermann, Paris, 1957.

51. G. Strang and G. Fix, Fourier analysis of the finite element method in Ritz-Galerkin Theory, Studies in Applied Mathematics, 48 (1969), 265-273. MR 41:2944

52. R. Strichartz, A Guide to Distribution Theory and Fourier Transforms, CRC Press, Ann Arbor, 1994. MR 95f:42001

53. R. Strichartz, Self-similarity on nilpotent Lie groups, Contemporary Mathematics, 140 (1992), 123-157. MR 94e:43011

54. M. E. Taylor, Noncommutative harmonic analysis, Mathematical Surveys, No. 22, American Mathematical Society, Providence, 1986. MR 88a:22021

55. F. Treves, F. Topological Vector Spaces, Distributions, and Kernels, Academic Press, New York, 1967. MR 37:726

56. V. S. Varadarajan, Lie Groups, Lie Algebras, and their Representations, Springer-Verlag, New York, 1984. MR 85e:22001 
57. L. F. Villemoes, Energy moments in time and frequency for two-scale difference equation solutions and wavelets, SIAM Journal of Mathematical Analysis, 23 (1992), 1519-1543. MR 94c:39002

58. K. Yoshida, Functional Analysis, Springer, New York, 1980.

Department of Mathematics, National University of Singapore, 2 Science Drive 2, SiNGAPORE 117543

E-mail address: wlawton@math.nus.edu.sg 